LA-UR- $98-1214$
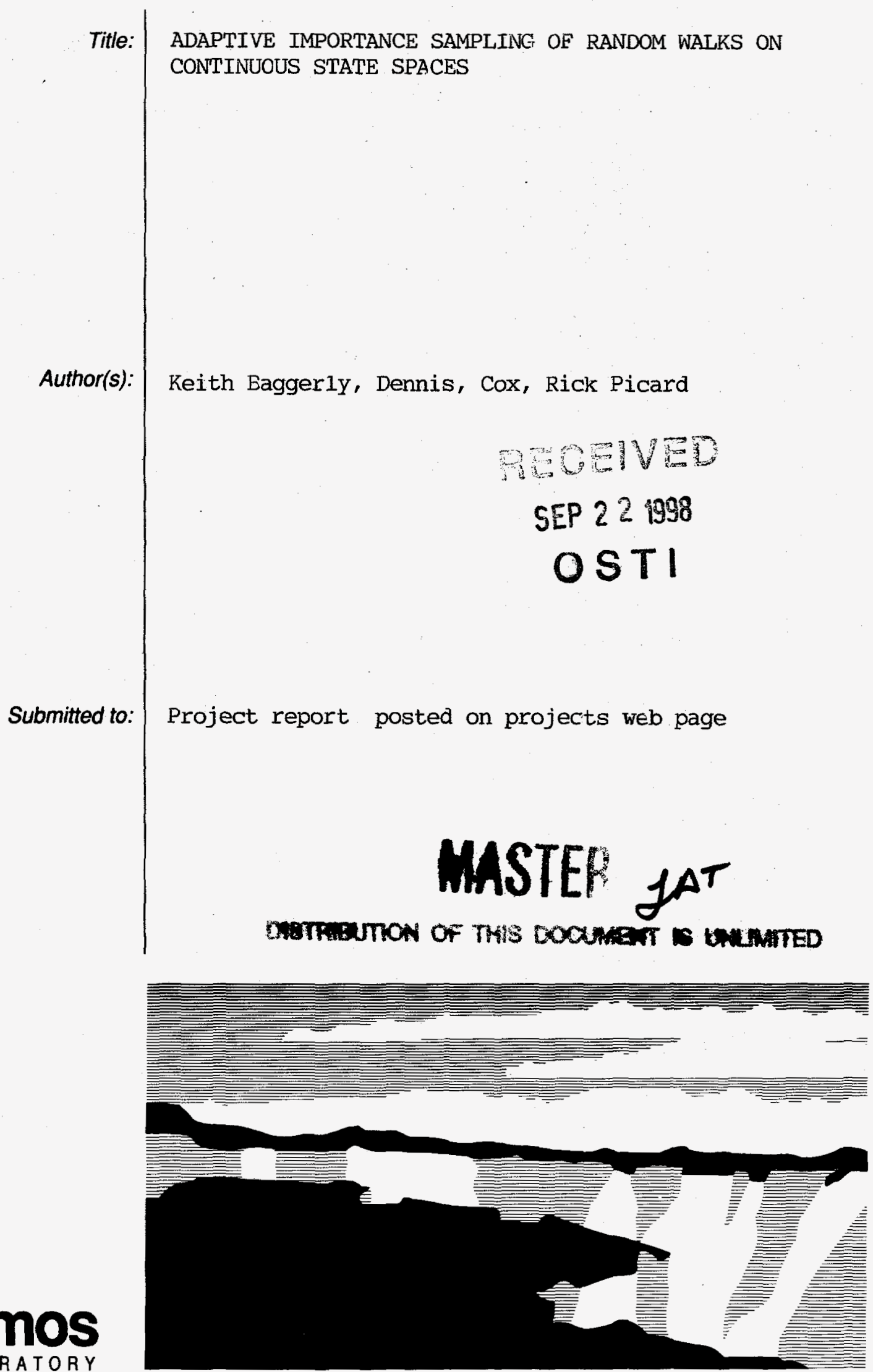

Los Alamos National Laboratory, an affirmative action/equal opportunity emplobyer, is operated by the University of California for the U.S. Department of Energy under contract W-7405-ENG-36. By acceptance of this article, the publisher recognizes that the U.S. Government retairs a nonexclusive, royalty-free license to publish or reproduce the published form of this contribution, or to allow others to do so, for U.S. Government purposes. The Los Alamos National Laboratory requests that the publisher identify this anticle as work performed under the auspices of the U.S. Department of Energy. 


\title{
Adaptive Importance Sampling for Random Walks on Continuous State Spaces ${ }^{1}$
}

\author{
Keith Baggerly, Dennis Cox, and Rick Picard
}

March 16, 1998

${ }^{1}$ Keith Baggerly and Dennis Cox are Assistant Professor and Professor, respectively, in the Statistics Department of Rice University, Houston, TX; and Rick Picard is a Technical Staff Member of the Statistics Group of Los Alamos National Laboratory, Los Alamos, NM. This research was supported as part of the Laboratory Directed Research and Development Program's ongoing effort to improve Monte Carlo methods. 


\section{DISCLAIMER}

This report was prepared as an account of work sponsored by an agency of the United States Government. Neither the United States Government nor any agency thereof, nor any of their employees, makes any warranty, express or implied, or assumes any legal liability or responsibility for the accuracy, completeness, or usefulness of any information, apparatus, product, or process disclosed, or represents that its use would not infringe privately owned rights. Reference herein to any specific commercial product, process, or service by trade name, trademark, manufacturer, or otherwise does not necessarily constitute or imply its endorsement, recommendation, or favoring by the United States Government or any agency thereof. The views and opinions of authors expressed herein do not necessarily state or reflect those of the United States Government or any agency thereof. 
The transition density is given below for all possible eventualities. Notationally, the ordered pair $\left(v_{i}, u_{i}\right)$ below denotes a state which is not $\Delta$. The equations, followed by explanatory text:

$$
\begin{aligned}
& p\left(\left(v_{0}, u_{0}\right),\left(v_{1}, u_{1}\right)\right)=\frac{c}{2} \sigma \exp \left[-\sigma\left(v_{1}-v_{0}\right)\right] \\
& \text { if } u_{0}=+1 \text { and } 0 \leq v_{0} \leq v_{1} \leq T \text {; } \\
& p\left(\left(v_{0}, u_{0}\right),\left(v_{1}, u_{1}\right)\right)=\frac{1}{2} \sigma \exp \left[-\sigma\left(v_{1}-v_{0}\right)\right] \\
& \text { if } u_{0}=+1 \text { and } 0 \leq v_{0} \leq T<v_{1} \text {; } \\
& p\left(\left(v_{0}, u_{0}\right),\left(v_{1}, u_{1}\right)\right)=\frac{c}{2} \sigma \exp \left[-\sigma\left(v_{0}-v_{1}\right)\right] \\
& \text { if } u_{0}=-1 \text { and } 0 \leq v_{1} \leq v_{0} \leq T \text {; } \\
& p\left(\left(v_{0}, u_{0}\right),\left(v_{1}, u_{1}\right)\right)=\frac{1}{2} \sigma \exp \left[-\sigma\left(v_{0}-v_{1}\right)\right] \\
& \text { if } u_{0}=-1 \text { and } v_{1}<0 \leq v_{0} \leq T \text {; } \\
& p\left(\left(v_{0}, u_{0}\right),\left(v_{1}, u_{1}\right)\right)=0 \quad \text { otherwise; } \\
& p\left(\left(v_{0}, u_{0}\right), \Delta\right)=(1-c)\left(1-e^{-\sigma\left(T-v_{0}\right)}\right) \\
& \text { if } 0 \leq v_{0} \leq T \text { and } u_{0}=+1 \text {; } \\
& p\left(\left(v_{0}, u_{0}\right), \Delta\right)=(1-c)\left(1-e^{-\sigma v_{0}}\right) \\
& \text { if } 0 \leq v_{0} \leq T \text { and } u_{0}=-1 \text {; } \\
& p\left(\left(v_{0}, u_{0}\right), \Delta\right)=1 \quad \text { if } v_{0}<0 \text { or } v_{0}>T ; \\
& p\left(\left(v_{0}, u_{0}\right), \Delta\right)=0 \text { otherwise; } \\
& p(\Delta, \Delta)=1 \text {. }
\end{aligned}
$$

To explain:

(1) Equations (1) and (2) correspond to an exponential step to the right (since the starting direction $u_{0}=+1$ ) for a particle originating from a location in the interval $[0, T]$. The factor of $1 / 2$ in both equations comes from the random direction of movement $u_{1}$. The factor $c$ in (1) is from nonabsorption (otherwise, 
the particle couldn't move to a state $\left.\left(v_{1}, u_{1}\right) \neq \Delta\right)$. Absorption can only occur if the particle stays in the interval $[0, T]$, which is the reason the transition density is different in the two regions $v_{1} \leq T$ and $v_{1}>T$. To explain (1) in detail, it is the probability of nonabsorption $c$ times the probability $1 / 2$ of moving either direction after the collision (note that $u_{1}$ is not specified; for either value \pm 1 we must account for the chance of that value) times the probability density function $\sigma \exp \left[-\sigma\left(v_{1}-v_{0}\right)\right]$ for the exponential step.

(2) Equations (3) and (4) are similar to equations (1) and (2) except the particle is moving to the left $\left(u_{0}=-1\right)$.

(3) Equation (5) simply states that there are no other allowed transitions between non-death states.

(4) Equations (6) and (7) correspond to absorption in the interval $[0, T]$. As the particle can only be absorbed if it does not escape, the absorption probability per collision (conditional on non-escape) of $1-c$ is multiplied by the probability of nonescape which is $1-e^{-\sigma\left(T-v_{0}\right)}$ or $1-e^{-\sigma v_{0}}$ for $u_{0}=+1$ and -1 , respectively.

(5) Equation (8) corresponds to death after escape.

(6) Equation (9) simply states that there are no other allowed transitions between non-death states and the death state, other than what has already been given.

(7) Equation (10) means the particle remains dead after it dies.

\subsection{Scoring.}

Recall that we wish to determine the probability of escape to the right. In the framework later defined in Section 3.1, this is expressed as the expected value or 
theoretical average value of a score function, namely the score function

$$
s_{\delta}((v, u), y)=\left\{\begin{array}{cl}
\delta & \text { if } v \leq T \text { and } y=\Delta \\
1+\delta & \text { if } v>T \text { and } y=\Delta \\
0 & \text { otherwise }
\end{array}\right.
$$

Here, $\delta>0$ is a constant chosen by the user, and is thus known. For a simulation of the analog random walk $X_{0}, X_{1}, \ldots$, the observed total score is

$$
\sum_{t} s_{\delta}\left(X_{t-1}, X_{t}\right)
$$

Note that a nonzero score for the rod problem is obtained only upon death, so there is only one nonzero term in the sum. This term is $1+\delta$ if death occurs after escape to the right, and is $\delta$ if death occurs by absorption or escape to the left. Death is certain to occur, in that the probability of remaining within the interval $[0, T]$ and avoiding absorption ad infinitum is zero. Upon death, we always collect the $\delta$ (sometimes this is referred to as the "life insurance"), and the expected value of the total score is thus $\delta$ plus the probability of escape to the right.

The mean total score for the particle history is

$$
\mu_{\delta}(x)=\delta+\mu_{0}(x)
$$

where $\mu_{0}(x)$ is the analog probability of escape to the right. Of course we are really interested in $\mu_{0}$ and one may wonder why we bother to add the life insurance $\delta$. It is added in for technical reasons discussed at greater length in Section 4. Basically, we want to ensure that the mean total score or importance function is strictly positive and bounded away from 0 . 


\subsection{A Parametric Model for the Expected Score.}

It is easy to see that for locations $v$ outside the rod we have

$$
\mu_{0}(v, u)= \begin{cases}1 & \text { if } v>T \\ 0 & \text { if } v<0\end{cases}
$$

The first line follows since if the particle has escaped to the right, it dies with certainty on the next step and scores 1 (when $\delta=0$ ), and the second line follows since if the particle escapes to the left it dies on the next step with no score (when $\delta=0$ ).

For $v$ a location in the interval $[0, T]$, the total mean score is different for particle movement to the right and left. In Section 6.1 we derive an exact expression for $\mu_{0}$. Importantly, the solution has the form

$$
\begin{aligned}
& \mu_{0}(v,+1)=\beta_{+1,1} e^{-\beta_{0} v}+\beta_{+1,2} e^{\beta_{0} v}, \\
& \mu_{0}(v,-1)=\beta_{-1,1} e^{-\beta_{0} v}+\beta_{-1,2} e^{\beta_{0} v},
\end{aligned}
$$

where

$$
\begin{aligned}
& \beta_{+1,1}=(\phi / 2)\left(1-\frac{1-c / 2}{\sqrt{1-c}}\right) \\
& \beta_{+1,2}=(\phi / 2)\left(1+\frac{1-c / 2}{\sqrt{1-c}}\right) \\
& \beta_{-1,1}=(\phi / 2) \frac{-c / 2}{\sqrt{1-c}} \\
& \beta_{-1,2}=(\phi / 2) \frac{c / 2}{\sqrt{1-c}}
\end{aligned}
$$

and

$$
\begin{aligned}
\beta_{0} & =\sigma(1-c)^{1 / 2} \\
\phi & =\left[\cosh \left(\beta_{0} T\right)+\beta_{0}^{-1} \sigma(1-c / 2) \sinh \left(\beta_{0} T\right)\right]^{-1},
\end{aligned}
$$

and sinh and cosh denote the hyperbolic sine and cosine, respectively. 
The existence of such a parametric model for the expected score allows the use of scores from a finite set of initial states to estimate the importance function everywhere. Curve fitting techniques may be used to construct such an estimate. The learning algorithm described in the next subsection iteratively improves the estimate of the importance function.

\subsection{Biasing the Random Walk.}

We can dramatically improve the Monte Carlo accuracy by simulating not the analog random walk but instead another random walk which "biases" the process to move in ways that tend to give higher scores. To correct for not simulating the analog random walk, we must "weight" the particle history. Suppose that we simulate from a random walk with transition density $q(x, y)$. Then the particle weight at step $t$ is

$$
L_{t}=\prod_{i=1}^{t} \frac{p\left(X_{i-1}, X_{i}\right)}{q\left(X_{i-1}, X_{i}\right)}
$$

The total weighted score is

$$
\sum_{t} s_{\delta}\left(X_{t-1}, X_{t}\right) L_{t}
$$

For the rod problem, there is only one nonzero term in the sum. Multiplying by the particle weight $L_{t}$ preserves the mean value - i.e., the expectation or theoretical average value of the total weighted score when simulating from the biased process with transition density $q(x, y)$ is exactly the same as the expectation of the total unweighted score when simulating the analog random walk.

As we shall see in Section 3.3, the mean total weighted score is exactly $\mu_{\delta}\left(x_{0}\right)$ if the process starts at $x_{0}$ and progresses according to the zero-variance transition density. This density $q_{\mu_{\delta}}(x, y)$ is proportional to the expected total score $s_{\delta}(x, y)+\mu_{\delta}(y)$ for a random walk which begins with a step from $x$ to $y$, and is given by

$$
q_{\mu_{\delta}}(x, y)=p(x, y) \frac{s_{\delta}(x, y)+\mu_{\delta}(y)}{\mu_{\delta}(x)} .
$$


Of course, computing this density requires knowing the expected score $\mu_{\delta}(x)$ for every $x$, which is what we are trying to determine, so this notion initially seems not very useful.

We can, however, simulate from the analog transition kernel $p(x, y)$ and then estimate $\mu_{\delta}(x)$. This leads to an approximate zero-variance $(A Z V)$ transition density

$$
q_{\hat{\mu}_{\delta}}(x, y)=p(x, y) \frac{s_{\delta}(x, y)+\hat{\mu}_{\delta}(y)}{\zeta_{\hat{\mu}}(x)},
$$

where $\hat{\mu}_{\delta}(x)$ is the estimate of $\mu_{\delta}(x)$ and $\zeta_{\hat{\mu}}(x)$ is the normalizing constant which makes $q_{\hat{\mu}_{\delta}}(x, y)$ a probability density function in $y$ (see (67)). Once a new estimate of $\mu_{\delta}$ is obtained, it can be substituted back into this form of the transition density, and the process can be iterated to convergence.

For the rod problem, we know $\mu_{\delta}$ (see (12) and (13) through (21)). For purposes of illustration, however, we assume we know only that $\mu_{\delta}$ has the parametric form that follows from (14) and (15) for states $(v, \pm 1)$ with $0 \leq v \leq T$, where the coefficients (or parameters) $\beta_{+1,1}, \beta_{+1,2}, \beta_{-1,1}$, and $\beta_{-1,2}$ are unknown (and $\beta_{0}$ has the value in (20)). We also know (13) gives $\mu_{\delta}$ outside of $[0, T]$. This then gives a parametric model for $\mu_{\delta}$, and the $\beta_{ \pm 1, j}, j=1,2$ are the parameters of the model. Estimation of $\mu_{\delta}$ reduces to estimating the parameters of the model.

In order for the particle weights in (22) to be defined and finite, we must have $p(x, y)>0$ only when $q_{\hat{\mu}_{\delta}}(x, y)>0$; in other words, if transition from a state $x$ to a state $y$ is possible for the analog random walk, it must also be possible for the importance sampling random walk. Otherwise, the importance sampling does not produce weighted scores having the right properties, which could conceivably lead to "false convergence" or to no convergence at all.

To ensure that random walks having positive probability under the analog random walk are also possible under the AZV random walks, estimates of $\mu=\mu_{\delta}$ for a given 
$\delta$ have the form

$$
\hat{\mu}(v, u)=\left\{\begin{array}{cl}
1+\delta & \text { if } v>T \\
\delta+\left(\hat{\beta}_{u, 1} e^{-\beta_{0} v}+\hat{\beta}_{u, 2} e^{+\beta_{0} v}\right)_{+} & \text {if } 0 \leq v \leq T \\
\delta & \text { if } v<0
\end{array}\right.
$$

where the parameter estimates $\hat{\beta}_{u, i}$ are obtained by least squares from the simulation data. Here, for $w$ a real number, $(w)_{+}$denotes the positive part function:

$$
(w)_{+}=\max \{0, w\}
$$

Also, the estimates $\hat{\beta}_{u, i}$ are based on scores from which $\delta$ has been subtracted - i.e., data which are unbiased when $\delta=0$. The role of the positive part function and the shifting by $\delta$ is to guarantee that $\hat{\mu}$ is bounded away from zero (in fact, $\hat{\mu} \geq \delta$ ), which might not happen otherwise because of the possibility of particle histories having zero scores (once $\delta$ has been subtracted) and the nature of least squares estimation being such that the estimated $\hat{\mu}$ could be negative at certain locations were it not for incorporation of the positive part function. Bounding $\hat{\mu}(v, u)$ away from zero provides protection that $q_{\hat{\mu}}(x, y)$ is positive whenever $p(x, y)>0$.

This protection comes at a (minor) cost: simulating random walks from such a transition kernel is nontrivial. Extensive details of the simulation for the rod problem are given in Section 6.2. This difficulty of simulating from the AZV random walk is an important practical consideration in other applications of the adaptive importance sampling procedure.

The value of $\delta$ to add to the score for the transition to the absorbing state $\Delta$ is somewhat arbitrary. Any (positive) value could be chosen, though large values of $\delta$ tend to produce inefficient results: this is perhaps most easily seen by inspection of the density $q_{\hat{\mu}}(x, y)$ of AZV random walk in $(24)$, in that

$$
\lim _{\delta \rightarrow \infty} q_{\hat{\mu}}(x, y) \rightarrow p(x, y)
$$


That is, when $\delta$ is very large, the AZV transition kernel strongly resembles the analog transition kernel, which typically is not compuationally efficient. Stated differently, as $\delta$ becomes large, the contributions $p(x, y) / q_{\hat{\mu}}(x, y)$ to particle weights tend towards 1.0 for all $x$ and $y$. Adjusting $\delta$ thus provides a means of "weight control" which can have considerable influence on the behavior of the simulation.

\subsection{Numerical Example.}

Consider the special case of the rod problem where the mean free path $\sigma=1$, the probability of absorption per collision is 0.1 (i.e., $c=0.9$ ), and the length of the rod is $T=20$ mean free paths. These parameter values are arbitrarily chosen as being nice round numbers and are such that a particle sourced at $v=0$ and moving to the right has probability $\mu_{0}(0,+1)$ of ultimately escaping the rod to the right equal to roughly 1 in 1000 . This example allows for a simple illustration of exponential convergence, where simulated results can be compared with a known solution.

The example also illustrates the difficulties of problems dealing with rare events, such as a the probability of a particle completely penetrating through shielding. In order to get accurate results from an analog simulation, a large number of particle histories must be simulated. Consider, for example, reducing uncertainties to a $1 \%$

relative error - that is, for the standard deviation $\left[\mu_{0}(0,+1)\left(1-\mu_{0}(0,+1)\right) / n\right]^{1 / 2}$ of the estimate from $n$ independent analog particle histories to be roughly $1 \%$ of the probability $\mu_{0}(0,+1)$ being estimated. To do this for the case where $\mu_{0}(0,+1)$ is roughly 0.001 , there would need to be roughly $n=10,000,000$ analog histories simulated from the initial state $(v, u)=(0,+1)$, the vast majority of these histories giving zero scores. This situation is clearly inefficient. Using adaptive importance sampling, essentially exact results (i.e., to machine computing accuracy) can be obtained from less than 2,000 histories. 
To implement the algorithm, a design $D$ and life insurance payoff $\delta$ are required. The design consists of a set of initial states $\left\{x_{1}, x_{2}, \ldots, x_{d}\right\}$ from which to simulate $r$ independent particle histories per state in the design. In other words, writing $x_{i}$ $=\left(v_{i}, u_{i}\right)$, in each iteration of the algorithm, we source particles from each initial location $v_{i}$ in the direction $u_{i}$. In situations where particle histories are expensive to simulate relative to the computational cost of updating importance sampling kernels, the number of initial states and number of replications should be kept relatively small.

To that end, consider the design where a particle is sourced inward from each end of the rod, $\left(v_{i}, u_{i}\right)=(0,+1)$ and $(20,-1)$ and 18 additional particles were sourced at intermediate states $\left\{\left(v_{i}, u_{i}\right)\right\}=(2, \pm 1),(4, \pm 1),(6, \pm 1),(8, \pm 1),(10, \pm 1),(12, \pm 1)$, $(14, \pm 1),(16, \pm 1)$, and $(18, \pm 1)$. In each iteration of the algorithm, $r=1$ particle history is simulated for each of the $d=20$ initial states in the design. Although interest lies solely in the penetration probability $\mu_{0}(0,+1)$, it is necessary to source particles from other initial states in order to obtain the zero-variance solution.

Also, the value $\delta=.01$ is arbitrarily chosen to bound the importance function away from zero for this example.

The algorithm requires an initial estimate $\hat{\mu}$ from which to obtain a transition density $q_{\hat{\mu}}$ for use in the first iteration. As with most iterative procedures, the closer the "starting value" $\hat{\mu}$ is to the actual solution $\mu$, the more quickly that convergence occurs. In some cases, a good estimate $\hat{\mu}$ may be available from experience with similar problems. When a good initial estimate is lacking, an alternative is to use the analog transition density $p(x, y)$ in the first iteration, which is the procedure used in here.

Figure 1 displays the results. Plotted using solid circles is the logarithm of the sum of squares $\sum_{i}\left(\hat{\mu}\left(x_{i}\right)-\mu\left(x_{i}\right)\right)^{2}$, where the sum is over the 20 design points. This metric corresponds to the metric of the main theorem. The roughly linear decrease in $\log$ scale reflects the exponential convergence of simulated particle histories to the 
zero-variance ideal. Once machine accuracy has been reached in the example, which takes about 90 iterations or a total of $(90)(20)=1,800$ simulated particle histories, there is no further decrease.

In practice, the "true" sum of squares, $\sum_{i}\left(\hat{\mu}\left(x_{i}\right)-\mu\left(x_{i}\right)\right)^{2}$, is unknown because the actual values $\mu\left(x_{i}\right)$ are unknown. Nonetheless, a close approximation to the plot of interest can be obtained by plotting the logarithm of the residual sum of squares $\sum_{i}\left(Y_{i, Q}-\hat{\mu}\left(x_{i}\right)\right)^{2}$, where $Y_{i, Q}$ denotes the score from the $i^{\prime}$ th design point when transition kernel $Q$ is used for the iteration, and again the sum is over the 20 design points. This residual sum of squares (the open circles in Figure 1) roughly tracks Figure 1, and can be used as a means of assessing convergence.

As a final note, even though interest is only in a single value of the mean total score, $\mu_{0}(0,+1)$ (equal to the probability that a particle sourced at $v=0$ and moving into the rod will completely penetrate the rod), the nature of the exponential convergence of the learning algorithm is such that it is achieved at all states $x$ or it is achieved nowhere. Thus, it is necessary that the design contain enough initial states for estimation of the importance function over the entire state space, and it is not sufficient for our algorithm to source particles from a single state of interest. 


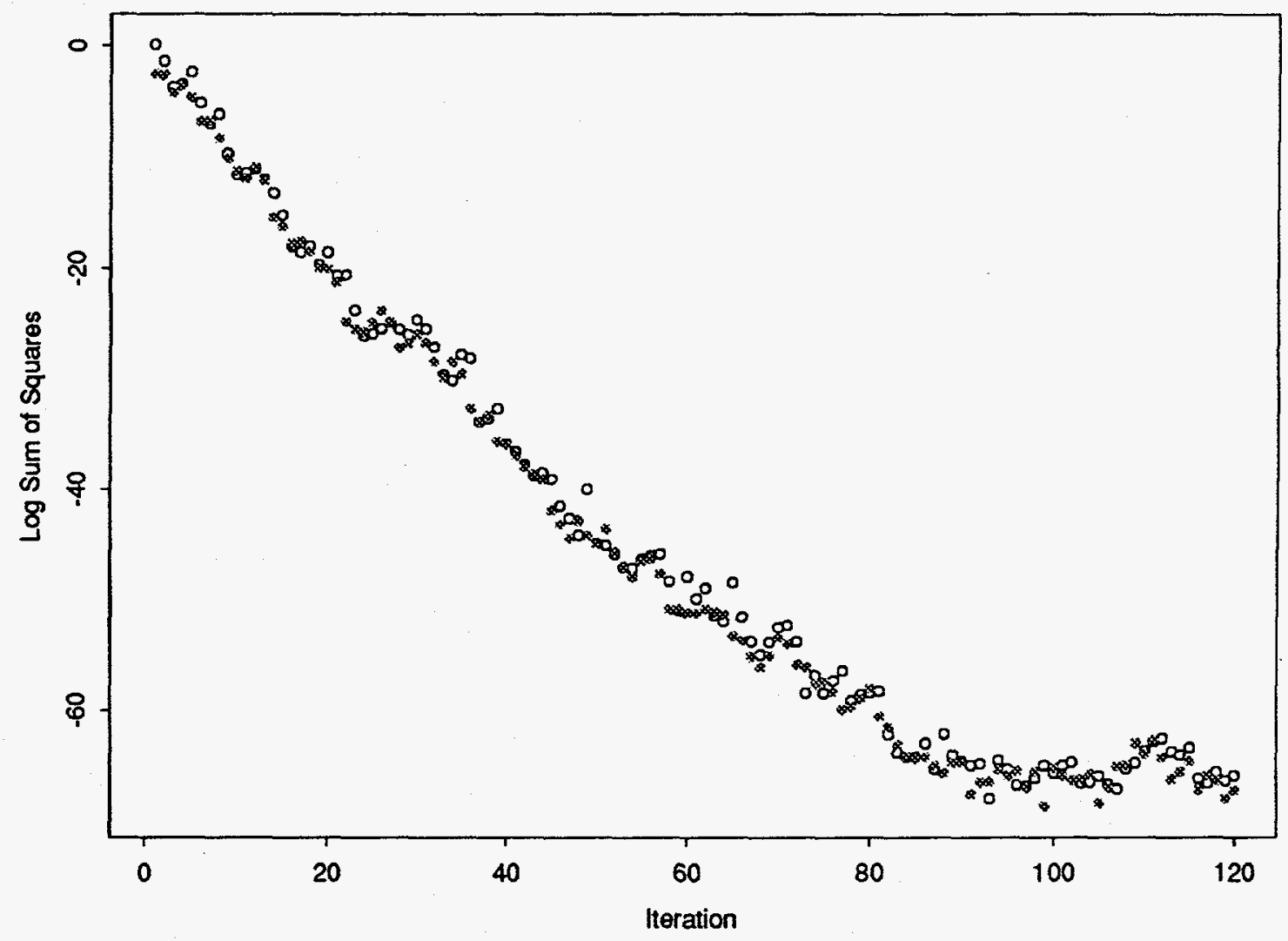

Figure 1: Exponential convergence for the Rod Problem: "true" sum of squares (closed circles) and residual sum of squares (open circles). 


\section{Notations, Definitions, and Basic Results.}

For purposes of exposition, the context of the general presentation remains that of a particle undergoing motion is some medium, possibly subject to collisions. We will illustrate basic concepts with the rod problem described in Section 2. However, the theory to follow is applicable to Markov chains of any origin. Although it is common to refer to the processes we are investigating as "random walks," in the jargon of probability theory a random walk is a special kind of Markov chain. In this section, we generally use the more formal terminology from probability theory.

The information on a particle at any particular stage of its history is called its state. It is necessary that all pertinent information be kept in the state - e.g. the position and momentum. For the rod problem, a state $x$ is of the form $(v, u)$ where $v$ is location and $u$ is direction. The collection of all possible states is referred to as the state space.

In general, the state space has an imbedded multidimensional structure. For example, in many particle transport problems, the state space is seven-dimensional: three dimensions for the particle's location, two dimensions (usually angles in a polar representation) for the particle's direction, one dimension for energy, and another dimension for time. As the multidimensional structure is not important to what follows, the simplified notation $x$ is used to denote a state.

The state of the random walk at step $t$ is denoted $X_{t}$. An entire particle history is denoted by $\left\langle X_{t}: t=0,1,2, \ldots\right\rangle$. The particle makes transitions from one state to another according to a conditional probability distribution known as the transition kernel. The transition kernel for the analog process is denoted $P(x, A)$, where $x$ denotes a state and $A$ denotes a set of states. Here, $P(x, A)$ is the probability that the particle makes a one-step transition from the state $x$ to a state in the set $A$, 
conditional on the particle being in $x$. Notationally,

$$
P(x, A)=\mathbf{P}\left[X_{t+1} \in A \mid X_{t}=x\right]
$$

There are two common types of transition kernels: discrete and continuous. In discrete cases, the set of states can be enumerated as a sequence and probabilities are computed by sums. For a discrete state space, we write

$$
P(x, A)=\sum_{y \in A} p(x, y)
$$

where

$$
p(x, y)=P(x,\{y\})
$$

i.e. $p(x, y)$ is the conditional probability of going from $x$ to $y$ in one step, given the particle is in the state $x$.

In the continuous setting, the state space is a continuum and $P(x,\{y\})=0$ for all states $x$ and $y$. Probabilities are computed by integration. In this case, the transition kernel is specified by a transition density function denoted $p(x, y)$ and

$$
P(x, A)=\int_{A} p(x, y) d y
$$

where $\int_{A}$ means integration over the set $A$. If the state space is multidimensional, then this is a multiple integral.

In the rod problem, the state space has both continuous and discrete elements. Recall that a state in this example is $(v, u)$ where $v$ is a location (in the continuum of real numbers) and $u$ is a discrete direction $( \pm 1)$. As an example of computing probabilities in the rod problem, suppose we wish to compute $P\left(\left(v_{0},+1\right), A\right)$ where $A=\left\{\left(v_{1}, u_{1}\right): a<v_{1}<b\right\}$ where $a$ and $b$ are given with $0<v_{0}<a<b<T$. That is, we are computing the probability that the next state is in the interval $(a, b)$ given that the particle is at $v_{0}$ and moving to the right. Note that the direction $u_{1}$ is not 
specified. Using (1), this is

$$
\begin{aligned}
P\left(\left(v_{0},+1\right), A\right) & =\int_{a}^{b} \sum_{u_{1}= \pm 1} p\left(\left(v_{0},+1\right),\left(v_{1}, u_{1}\right)\right) d v_{1} \\
& =\int_{a}^{b} c \sigma \exp \left[-\sigma\left(v_{1}-v_{0}\right)\right] d v_{1}
\end{aligned}
$$

In the first expression, we integrate over the continuous variable $v_{1}$ and sum over the discrete variable $u_{1}$.

Mathematical rigor requires the use of measure theory for a completely general formulation. To illustrate the concepts, the discrete and continuous cases are often referenced, and it is noted that measure theory incorporates both settings (and more - e.g., the rod problem).

\subsection{Formal Definitions.}

Let $E$ denote the state space and $\mathcal{E}$ the $\sigma$-field of measurable sets (it is not intended that the reader be well versed in measure theory to follow the presentation, but those interested in such details can consult standard texts, such as Royden 1968). In general, we do not include the cemetery $\Delta$ in the state space $E$. Further discussion on $\Delta$ is given in Subsection 3.1.1 below. The state space for the rod problem is $E=$ $\mathbb{R} \times\{-1,+1\}$, where $\mathbb{R}$ is the collection of real numbers, and the Cartesian product notation " $\times$ " means the states are ordered pairs $(v, u)$ with $v$ in $\mathbb{R}$ and $u$ in $\{-1,+1\}$. The measurable sets in the rod problem may be thought of as the collection of all subsets of the state space. This is technically not correct (nonmeasurable sets do exist), but we will pretend for present purposes that all subsets referenced herein are measurable in all state spaces and not worry further about this technical detail.

As described above, a general Markov chain is defined through its transition kernel $P: E \times \mathcal{E} \longrightarrow[0,1]$, Formally, the transition kernel $P(x, \cdot)$ is a measure for each $x$. We generally place the measure with respect to which the integral is being taken 
directly after the integral sign. This is convenient for many formulae in Markov chain theory. Thus, for a real valued function $h(x)$,

$$
P h(x)=\int P(x, d y) h(y)
$$

Here $P$ is the transition operator. It is an integral operator on functions $h: E \longrightarrow \mathbb{R}$ with integral kernel $P(x, d y)$.

The two most common settings are of course are the discrete and continuous transition kernels already discussed.

Discrete $E: E$ is finite or countably infinite, $p(x, y)$ is the conditional probability of going from $x$ to $y$, and the transition kernel is identified with a transition probability matrix. In this case, integrals with respect to $P(x, \cdot)$ are sums, i.e.

$$
\int P(x, d y) h(y)=\sum_{y \in E} p(x, y) h(y)
$$

In the matrix representation of Section 2.3 of Karlin and Taylor (1975), $p(x, y)$ is the $(x, y)$ entry of the transition probability matrix.

Continuous $E: E$ is a subset of a Euclidean space, and $p(x, y)$ is the conditional density of going from $x$ to $y$. In this case, integrals with respect to $P(x, \cdot)$ may be written as "ordinary" (Riemann or Lebesgue) integrals as in

$$
\int P(x, d y) h(y)=\int_{E} p(x, y) h(y) d y
$$

For both the discrete and continuous cases, we refer to $p(x, y)$ as the transition density. The $n$-step transition kernel denoted $P^{n}$. In words, $P^{n}(x, A)$ is the probability of going from $x$ to a state in $A$ in exactly $n$ steps, i.e.

$$
P^{n}(x, A)=\mathbf{P}\left[X_{t+n} \in A \mid X_{t}=x\right]
$$


It can be defined inductively by

$$
\begin{aligned}
P^{1}(x, A) & =P(x, A), \\
P^{n+1}(x, A) & =\int_{\bar{E}} P(x, d y) P^{n}(y, A)=\int_{\bar{E}} P^{n}(x, d y) P(y, A) .
\end{aligned}
$$

For completeness, we also need to note that $P^{0}(x, \cdot)$ is a unit point mass (or Dirac delta function) at $x$, i.e.

$$
P^{0}(x, A)= \begin{cases}0 & \text { if } x \notin A, \\ 1 & \text { if } x \in A .\end{cases}
$$

Note that as an integral operator, $P^{0}$ is the identity:

$$
P^{0} h(x)=h(x)
$$

For finite state spaces, where $P$ corresponds to a transition probability matrix, the $n$-step transitions can be extracted from the $n$ 'th power of the transition probability matrix (e.g. Karlin and Taylor 1975, Theorem 3.1). More generally, a messier but perhaps clearer definition of $P^{n}$ is

$$
P^{n}(x, A)=\int_{A} P\left(y_{n-1}, d y_{n}\right) \int_{E} P\left(y_{n-2}, d y_{n-1}\right) \cdots \int_{\bar{E}} P\left(y_{1}, d y_{2}\right) \int_{\bar{E}} P\left(x, d y_{1}\right) .
$$

Note that the order in which the integrals are computed is from right to left (which is the correct order when they are treated as operators on the integrand).

The overarching probability measure for a history is denoted $\mathbf{P}_{x, P}$ where $x$ is the starting value (i.e. $X_{0}=x$ ). Note that the subscript $x$ indicates the starting value for that particle history and the subscript $P$ indicates the transition kernel used to generate the history. The corresponding mathematical expectation operator (theoretical average or mean) is denoted $\mathrm{E}_{x, P}$. Complicated probabilities which depend on the entire history can usually be expressed in terms of the transition kernel. For 
example, if $A$ is a subset of $E$, then the probability that the particle ever visits $A$ given that the starting value $X_{0}=x$ is computed by

$$
\begin{aligned}
\mathbf{P}_{x, P} & {\left[X_{t} \in A \text { for some } t \mid X_{0}=x\right] } \\
& =\sum_{t=0}^{\infty} \mathbf{P}_{x, P}\left[X_{s} \in A^{c}, 0 \leq s<t, \text { and } X_{t} \in A \mid X_{0}=x\right] \\
& =\sum_{t=0}^{\infty} \int_{A} P\left(y_{t-1}, d y_{t}\right) \int_{A^{c}} P\left(y_{t-2}, d y_{t-1}\right) \cdots \int_{A^{c}} P\left(y_{1}, d y_{2}\right) \int_{A^{c}} P\left(x, d y_{1}\right)
\end{aligned}
$$

Expression (28) is the formal representation of the probability that the particle ever visits the set $A$ - i.e., that there is some step number $t$ such that $X_{t}$ is in $A$. In (29), we have decomposed the event by considering the first step the particle enters $A$. We use the notation $A^{c}$ to denote the complement of $A$. Thus, $X s \in A^{c}$, $0 \leq s<t$, means that the particle is not in $A$ before step number $t$. Thus, $\left[X_{s} \in A^{c}, 0 \leq s<t\right.$, and $\left.X_{t} \in A\right]$ is the event that the particle enters the set $A$ for the

first time on step $t$. Finally, (30) gives integral expressions using the transition kernel for the probabilities in (29). As another example using the expectation operator $\mathbf{E}_{x, P}$ :

$$
P h(x)=\mathrm{E}_{x, P}\left[h\left(X_{1}\right)\right]
$$

We will shortly consider probabilities and expectations for other Markov chains with different transition kernels, which is why we introduce the transition kernel as a subscript to the probability measure and expectation operator.

\subsubsection{Transience and the Cemetery.}

In keeping with applications of interest, it is assumed that the chain is transient, i.e. the particle eventually escapes or is absorbed. To accommodate transience, we create a state $\Delta \notin E$ called the death state or cemetery. The cemetery is absorbing in that there is no life after death, i.e.

$$
\mathbf{P}_{x, P}\left[X_{t+1}=\Delta \mid X_{t}=\Delta\right]=1
$$


and transience means that death eventually occurs with certainty, i.e.

$$
\mathbf{P}_{x, P}\left[\exists \tau<\infty, X_{t}=\Delta, \forall t \geq \tau\right]=1, \quad \forall x \in E
$$

Here, $\tau$ is the step at which death occurs, i.e.

$$
\tau=\min \left\{t: X_{t}=\Delta\right\}
$$

Denote $\bar{E}=E \cup\{\Delta\}$ and extend $P$ to $\bar{E}$ by defining $P(x,\{\Delta\})=1-P(x, E)$.

Continuous Case: Because transitions to the death state have a discrete "lump" of probability, there is an extra term in all of the integrals, viz.

$$
\int_{\bar{E}} P(x, d y) h(x, y)=p(x, \Delta) h(x, \Delta)+\int_{E} p(x, y) h(x, y) d y
$$

\subsubsection{Scoring}

With each step of the random walk, a score is accumulated. These scores correspond to some physical quantity of interest and the objective of the simulation is to estimate the expected score. Some simple examples include estimating the proportion of particles emitted from a source that penetrate a shield, estimating the energy release within a specified region, estimating the proportion of particles entering a location that are in a specified energy range, and estimating the probability that a given event occurs before a specified amount of time has elapsed.

Mathematically, then, the objective is to estimate

$$
\mu(x)=\mathbf{E}_{x, P}\left[\sum_{t=1}^{\tau} s\left(X_{t-1}, X_{t}\right)\right]
$$

where $s: E \times \bar{E} \longrightarrow[0, \infty)$ is a given score function. That is, $s\left(X_{t-1}, X_{t}\right)$ is the score accumulated for the individual transition from $X_{t-1}$ to $X_{t}$ at step $t$, and such scores are added over the particle history. Thus, $\mu(x)$ is the expected total score for a random walk starting at $x$. Sometimes, $\mu(x)$ is called the importance function. The 
upper limit of the summation can be extended to $\infty$ in the preceding equation by adopting the convention

$$
s(\Delta, x)=0, \quad \forall x \in \bar{E},
$$

and then

$$
\mu(\Delta)=0 .
$$

We usually abide by the convention that functions $h$ defined on $E$ extend to $\bar{E}$ by $h(\Delta)=0$, so $\mu(\Delta)=0$ conforms with this convention. Care is needed when $s(x, \cdot)$ does not satisfy $s(x, \Delta)=0$ necessarily, so the "convention" must be constantly checked.

Conditioning on the first step and using the Markov property, we have

$$
\begin{aligned}
\mu(x) & =\mathbf{E}_{x, P}\left[\sum_{t=1}^{\tau} s\left(X_{t-1}, X_{t}\right)\right] \\
& =\mathbf{E}_{x, P}\left\{s\left(x, X_{1}\right)\right\}+\mathbf{E}_{x, P}\left\{\sum_{t=2}^{\tau} s\left(X_{t-1}, X_{t}\right)\right\} \\
& =\mathbf{E}_{x, P}\left\{s\left(x, X_{1}\right)\right\}+\mathbf{E}_{x, P}\left\{\mathbf{E}_{x, P}\left[\sum_{t=2}^{\tau} s\left(X_{t-1}, X_{t}\right) \mid X_{1}\right]\right\} \\
& =\mathbf{E}_{x, P}\left\{s\left(x, X_{1}\right)\right\}+\mathbf{E}_{x, P}\left\{\mu\left(X_{1}\right)\right\}
\end{aligned}
$$

To explain, in (35) we are simply separating the score from the first transition. Equation (36) follows because the unconditional expectation of a conditional expectation is the same as the unconditional expectation of the random variable initially. In (36), we are using the Markov property: given that the particle is at $X_{1}$ after the first step, it doesn't matter what happened at the 0 'th step - we expect a total score of $\mu\left(X_{1}\right)$.

We can rewrite this important recursion relation in terms of the transition kernel:

$$
\mu(x)=\int_{E} P(x, d y)[s(x, y)+\mu(y)],
$$

which gives an integral equation for $\mu$ (or linear system of equations for a discrete state space). 
Continuous Case: The preceding equation (38) becomes

$$
\mu(x)=s(x, \Delta) p(x, \Delta)+\int_{E}[s(x, y)+\mu(y)] p(x, y) d y .
$$

For the rod problem, (38) is the basis for a coupled system of integral equations, which can be solved to give the parametric model for the expected score $\mu(x)$. The solution is given in Section 6.1, Equations (94) to (98).

\subsection{Importance Sampling.}

The most straightforward way to estimate $\mu(x)$ is to simulate many particle histories using the analog transition kernel starting at the state $x$, and average the corresponding total scores. As noted in the Introduction, however, simulating from the analog chain is often inefficient, as the variance of the estimate can be large. This is especially true when dealing with rare events.

Thus we consider estimation of $\mu(x)$ by simulating from another chain with transition kernel $Q(x, A)$. The kernel $Q$ is referred to as the importance sampling kernel and its associated Markov chain as the importance sampling chain, while the original $P$ and its chain are called the analog kernel and analog chain. Usually, $Q$ is chosen to "bias" random walks to preferentially favor those histories whose scores are large, thereby improving overall estimation. Of course, we must adjust for using the biased random walk. This is accomplished by "weighting" the scores, as discussed in some detail below.

First, a technical definition. If $P_{1}$ and $P_{2}$ are two probability measures, we say that $P_{2}$ dominates $P_{1}$ if there is a real valued function $g(x)$ such that for any other real valued function $h(x)$,

$$
\int_{E} P_{1}(d x) h(x)=\int_{E} P_{2}(d x) g(x) h(x) .
$$


The function $g(x)$ here is called the Radon-Nikodym derivative of $P_{1}$ with respect to $P_{2}$. The Radon-Nikodym theorem states that $P_{2}$ dominates $P_{1}$ if and only if the following condition holds:

$$
P_{2}(A)=0 \text { implies } P_{1}(A)=0 .
$$

Operationally, we write (39) by dropping the integrals and the arbitrary function $h(x)$ :

$$
P_{1}(d x)=P_{2}(d x) g(x)
$$

Alternatively, we write

$$
\frac{P_{1}(d x)}{P_{2}(d x)}=g(x) .
$$

Sometimes, the use of Radon-Nikodym derivatives is referred to as a "change of measure," i.e. we can change one measure to another in an integral, provided we correct by multiplying by the Radon-Nikodym derivative. A good reference for Radon-Nikodym theory is Royden (1968).

We briefly review classical importance sampling and show that Radon-Nikodym theory is fundamental to this subject. Suppose we wish to estimate the integral $\int P_{1}(d x) h(x)$. If we can simulate $X_{1}, \ldots, X_{n}$ from the probability distribution $P_{1}$, then the sample average $(1 / n) \sum_{i=1}^{n} h\left(X_{i}\right)$ is an unbiased estimate of $\int P_{1}(d x) h(x)$. On the other hand, if $P_{2}$ dominates $P_{1}$ and the Radon-Nikodym derivative is $g(x)$, then we can simulate $Y_{1}, \ldots, Y_{n}$ from $P_{2}$ and the sample average $(1 / n) \sum_{i=1}^{n} h\left(Y_{i}\right) g\left(Y_{i}\right)$ is an unbiased estimate of $\int_{E} P_{2}(d x) g(x) h(x)=\int P_{1}(d x) h(x)$. Note that now the "score" $h\left(Y_{i}\right)$ is "weighted" by the Radon-Nikodym derivative $g\left(Y_{i}\right)$ to correct for having simulated from a "biased" distribution.

Assumption 3.1 To be a valid importance sampling kernel for $P$, the kernel $Q$ must satisfy:

(i) $E$ is transient under $Q$; 
(ii) $Q(x, \cdot)$ dominates $P(x, \cdot)$ for all $x \in \bar{E}$.

In condition (i), the term transient means that death occurs with probability one under $Q$, as formalized in (32). Mathematically,

$$
\mathbf{P}_{x, Q}\left[\exists \tau<\infty, X_{t}=\Delta, \forall t \geq \tau\right]=1, \quad \forall x \in E
$$

Note that the subscript $Q$ appears on the probability $\mathbf{P}_{x, Q}$. The requirement that the random walk under $Q$ is transient is a technical one we need for the theory that follows. From the Radon-Nikodym theorem (see (40) above), condition (ii) means the same as

For all subsets $A$ of $E$ and all states $x$ in $E$, if $Q(x, A)=0$, then $P(x, A)=0$.

Loosely speaking, for the common setting where we have transition densities, this means

$$
q(x, y)=0 \text { implies } p(x, y)=0 .
$$

This condition allows us to apply Radon-Nikodym theory so that integrals with respect to $P(x, \cdot)$ can be expressed in terms of integrals with respect to $Q(x, \cdot)$. We denote the Radon-Nikodym derivative $\ell_{1}(x, y)$. Thus, using (41) we write

$$
P(x, d y)=Q(x, d y) \ell_{1}(x, y)
$$

The defining property of Radon-Nikodym derivatives from (39) applied to this situation is

$$
\int P(x, d y) h(y)=\int Q(x, d y) \ell_{1}(x, y) h(y) .
$$

Assumption (ii) is what makes importance sampling possible: we can unbiasedly estimate integrals under $P(x, \cdot)$ by sampling from $Q(x, \cdot)$ and "correcting" by multiplying in the weight $\ell_{1}(x, \cdot)$. Of course, this is only meaningful so far for a single step. The 